\title{
Interpretable clinical prediction via attention-based neural network
}

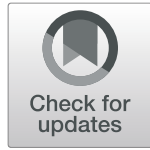

\author{
Peipei Chen ${ }^{1,2}$, Wei Dong ${ }^{3}$, Jinliang Wang ${ }^{4}$, Xudong Lu ${ }^{1,2}$, Uzay Kaymak ${ }^{2,1}$ and Zhengxing Huang ${ }^{1 *}$ \\ From 5th China Health Information Processing Conference \\ Guangzhou, China. 22-24 November 2019
}

\begin{abstract}
Background: The interpretability of results predicted by the machine learning models is vital, especially in the critical fields like healthcare. With the increasingly adoption of electronic healthcare records (EHR) by the medical organizations in the last decade, which accumulated abundant electronic patient data, neural networks or deep learning techniques are gradually being applied to clinical tasks by utilizing the huge potential of EHR data. However, typical deep learning models are black-boxes, which are not transparent and the prediction outcomes of which are difficult to interpret.

Methods: To remedy this limitation, we propose an attention neural network model for interpretable clinical prediction. In detail, the proposed model employs an attention mechanism to capture critical/essential features with their attention signals on the prediction results, such that the predictions generated by the neural network model can be interpretable.

Results: We evaluate our proposed model on a real-world clinical dataset consisting of 736 samples to predict readmissions for heart failure patients. The performance of the proposed model achieved 66.7 and $69.1 \%$ in terms of accuracy and AUC, respectively, and outperformed the baseline models. Besides, we displayed patient-specific attention weights, which can not only help clinicians understand the prediction outcomes, but also assist them to select individualized treatment strategies or intervention plans.
\end{abstract}

Conclusions: The experimental results demonstrate that the proposed model can improve both the prediction performance and interpretability by equipping the model with an attention mechanism.

Keywords: Interpretability, Attention mechanism, Clinical prediction, Deep learning

\section{Background}

Recently, deep learning techniques have achieved great success in healthcare domain due to their impressive performance [1-3]. Specifically, with the increasingly adoption of electronic healthcare records (EHR) by the medical organizations in the last decade, a large volume of electronic patient data is accumulated, and thereafter

\footnotetext{
* Correspondence: zhengxinghuang@zju.edu.cn

${ }^{1}$ College of Biomedical Engineering and Instrumental Science, Zhejiang University, Hangzhou, China

Full list of author information is available at the end of the article
}

neural networks or deep learning techniques are gradually being applied to clinical prediction tasks by utilizing the huge potential of EHR data, e.g. clinical risk assessment, outcome prediction, treatment effect estimation and treatment recommendations [4-7].

However, typical deep learning models are blackboxes, which are not transparent and the prediction outcomes of which are difficult to interpret [8]. Therefore, although deep learning models have shown remarkable performance on most clinical prediction tasks, the lack

(c) The Author(s). 2020 Open Access This article is licensed under a Creative Commons Attribution 4.0 International License, which permits use, sharing, adaptation, distribution and reproduction in any medium or format, as long as you give appropriate credit to the original author(s) and the source, provide a link to the Creative Commons licence, and indicate if changes were made. The images or other third party material in this article are included in the article's Creative Commons licence, unless indicated otherwise in a credit line to the material. If material is not included in the article's Creative Commons licence and your intended use is not permitted by statutory regulation or exceeds the permitted use, you will need to obtain permission directly from the copyright holder. To view a copy of this licence, visit http://creativecommons.org/licenses/by/4.0/ The Creative Commons Public Domain Dedication waiver (http://creativecommons.org/publicdomain/zero/1.0/) applies to the data made available in this article, unless otherwise stated in a credit line to the data. 
of interpretability makes them difficult to be practically adopted in the real clinical settings.

The interpretability is vital for the successful applications of machine learning models in the healthcare domain [9]. The reasons for the requirement of interpretable models are multiple, as indicated in literature $[10,11]$. Firstly, interpretability is the prerequisite for trust [12]. Healthcare professionals tend to have more confidence in the models which are well-understood or the models which can provide explanations. Secondly, interpretable models are expected to provide useful information to healthcare professionals and assist them to make decisions [13]. For example, instance-based models can support clinicians to take actions by pointing to similar patients. Additionally, interpretable models can help healthcare professionals gain insights into new knowledge [14]. There are also other reasons for the requirement of interpretability, such as legislation (the right to explanation in EU's GDPR (General Data Protection Regulation)) [15], reducing bias and capturing causality [16].

In this study, we present an attention based neural network model to improve the interpretability of the clinical predictions. We evaluated our model on a realworld EHR dataset to predict the readmissions of heart failure patients. The experimental results demonstrate that our proposed model can not only improve the prediction performance but also provide interpretations on the prediction results.

\section{Related works}

In this section, we briefly review the existing interpretable models or techniques enabling interpretations for black-box deep learning models, which can be broadly classified into two categories [10].

The first category relates to transparency of the model (i.e. how does the model work?). A transparent model can be understood at the level of the model itself, i.e. mechanistic or algorithmic transparency [17]. Linear model or logistic regression, decision tree and rule based models (e.g. fuzzy inference system [18]) are commonly considered to be transparent [8]. For example, the coefficients of the linear model could be interpreted as the strengths of the relationship between each feature and the label, and the sign of each coefficient indicates the direction of the relationship. However, such models become less interpretable when the models are too complex, e.g. deep decision trees and unmanageable number of rules [10].

The second category comprises various techniques which can provide post-hoc explanations for the blackbox models. In contrast to the intrinsically transparent models in the first category mentioned above, the posthoc interpretability may not attempt to interpret the inner work of the model, but seek to explain the predictions of the opaque or black-box model without sacrificing the performance $[8,10]$. The popular techniques of post-hoc interpretations contain explanations by text (natural language), explanations by visualization, explanations by a surrogate transparent model, and explanations by attention mechanism, as briefly discussed below:

Text explanations can provide qualitative understanding of the model predictions by presenting human understandable verbal words. One approach is to train two models simultaneously, one for prediction and another to generate textual explanations. For example, McAuley and Leskovec [19] presented a model to recommend products by simultaneously training a latent factor model for rating prediction and a topic model for textual product reviews. The predicted ratings can be explained by the top words in the topics.

Visualization explanations (e.g. heat maps) can provide post-hoc interpretations by visualizing what the model learned. For instance, t-SNE (t-Distributed Stochastic Neighbor Embedding) is commonly exploited to visualize the learned high-dimensional representations in 2D space [20].

Explanations by surrogate model improve the interpretability of black-box models by interpreting the source opaque model utilizing a transparent surrogate model (e.g. linear model, logistic regression, decision tree, instance-based model or rule-based model). For example, after training a deep learning model, we can identify the most similar patients to the source patient based on the learned latent representations to justify the model prediction $[21,22]$. In addition, LIME (Local Interpretable Model-agnostic Explanations) [11] explain the predictions of an opaque model by approximating it locally with an interpretable model, e.g. learning an interpretable model locally around the prediction.

Attention explanations are recently advocated to open a new window for interpreting deep learning models. Originally, the attention mechanism is mainly used to model dependencies between sequences regardless of their actual distances [23, 24]. It has achieved great success in many sequence modeling tasks, e.g. neural machine translation [23] and speech recognition [25]. Recently, attention mechanisms are increasingly applied to improve not only the accuracy but also the interpretability of deep learning models [26-28]. In [26], the authors proposed the GRaph-based Attention Model (GRAM) for healthcare representation learning, which infuses information from medical ontologies into deep learning models via attention mechanism and the attention behavior during prediction could be explained intuitively by showing the attention weights of each node in the knowledge graph. Choi et.al [27] proposed a model 


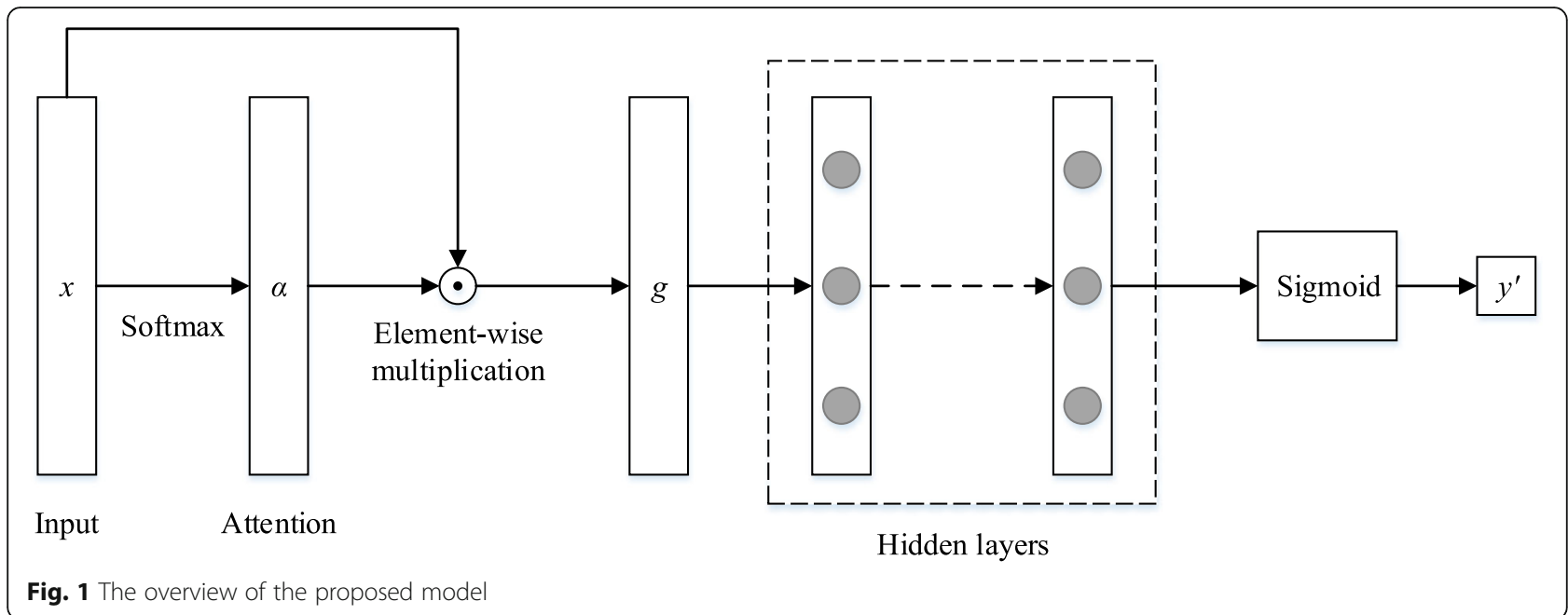

known as RETAIN, a two-level neural attention model for sequential data, which provides detailed interpretation of the prediction results while retaining the prediction accuracy comparable to RNN. In RETAIN, when keeping the attention fixed, the model prediction can be interpreted by analyzing the changes of each label in relation to changes in the original inputs, i.e. the input variable that yields the largest change in label will be the input variable with highest contribution.

Along with this direction, this study proposes an interpretable neural model equipped with an attention mechanism to address the clinical prediction problem, which can provide patient-specific attention weights on features such that the prediction results can be explained.

\section{Methods}

In this section, we firstly introduce the problem definition and notations used in this paper, and then present our proposed model in detail.

\section{Problem definition}

In this paper, the dataset is extracted from a large amount of EHR. A particular patient sample contains $m$ features (characteristics) and is usually represented as a feature vector $x$. The dataset consisting of $n$ patient samples can be represented as a matrix:

$$
X=\left[x_{1}, \cdots, x_{n}\right]=\left[\begin{array}{ccc}
x_{11} & \cdots & x_{n 1} \\
\vdots & \ddots & \vdots \\
x_{1 m} & \cdots & x_{n m}
\end{array}\right]
$$

Let $Y$ be the labels (i.e. readmitted/non readmitted) of the $n$ patient samples and can be denoted as:

$$
Y=\left[y_{1}, \cdots, y_{n}\right]
$$

The goal of this study is to predict the labels of the patients based on the patient characteristics in an interpretable manner.

\section{The proposed model}

Figure 1 depicts the overview of the proposed attentionbased model (we denote it as MLP_attention). The core

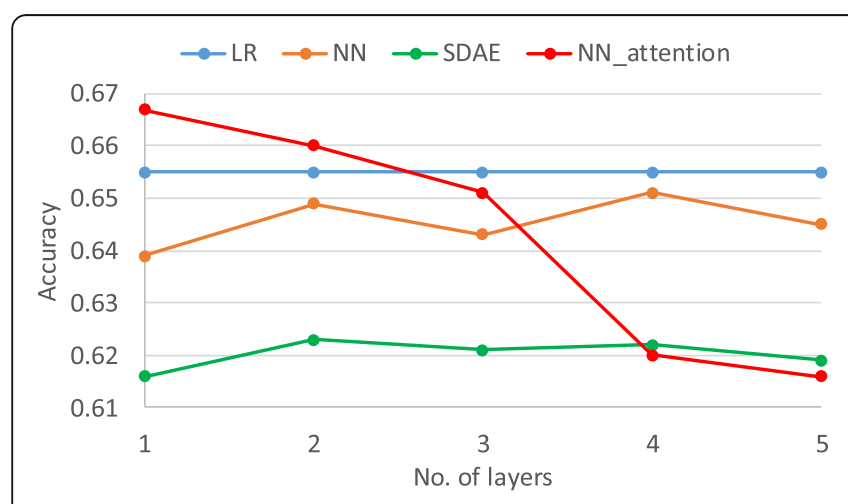

(a)

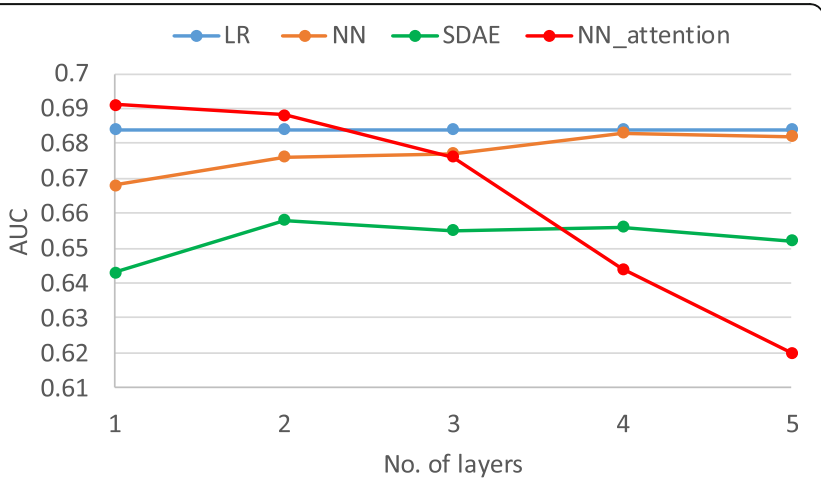

(b)

Fig. 2 The (a) Accuracy and (b) AUC of the proposed model with different number of hidden layers in comparison with baseline models 
Table 1 The prediction performance of all the models (mean \pm std. (standard deviation))

\begin{tabular}{llllll}
\hline Models & Accuracy & Precision & Recall & F1 & AUC \\
\hline MLP_attention & $\mathbf{0 . 6 6 7} \pm \mathbf{0 . 0 3 0}$ & $\mathbf{0 . 7 1 0} \pm \mathbf{0 . 0 2 0}$ & $0.795 \pm 0.059$ & $\mathbf{0 . 7 4 9} \pm \mathbf{0 . 0 2 9}$ & $\mathbf{0 . 6 9 1} \pm \mathbf{0 . 0 4 7}$ \\
MLP & $0.651 \pm 0.028$ & $0.692 \pm 0.022$ & $\mathbf{0 . 7 9 9} \pm \mathbf{0 . 0 6 2}$ & $0.741 \pm 0.030$ & $0.683 \pm 0.041$ \\
LR & $0.655 \pm 0.027$ & $0.700 \pm 0.019$ & $0.792 \pm 0.043$ & $0.743 \pm 0.024$ & $0.684 \pm 0.039$ \\
SDAE & $0.623 \pm 0.025$ & $0.670 \pm 0.018$ & $0.782 \pm 0.038$ & $0.722 \pm 0.022$ & $0.658 \pm 0.033$ \\
\hline
\end{tabular}

idea of the proposed model is to use an attention mechanism to capture the contribution of each input patient feature to the prediction, so that the generated prediction results can be interpreted. We introduce our proposed model below in detail.

Given the inputs $x$, we firstly feed $x$ into a fully connected layer (with Softmax function as the activation function) to generate the attention signals $\alpha$ (see Eq. (3)). The number of output nodes of the fully connected layer is the same with the length of $x$, i.e. $m$. The Softmax function ensures that the sum of the attention weights of all patient features equals to one.

$$
\alpha=\text { Soft } \max (W x+b)
$$

Then, we obtain the final input representation $g$ by the element-wise multiplication of the inputs $x$ and the generated attention signals $\alpha$ :

$$
g=\alpha \odot x
$$

where $\odot$ stands for the element-wise multiplication.

With the final input representation $g$, a multilayer perceptron (MLP) model with several hidden layers is used to predict the labels. The function of the output layer is Sigmoid function. We select 0.5 as the threshold to obtain the predicted labels.

We train the attention layer together with MLP. The loss function of the proposed model is defined as the follows:

$$
\begin{aligned}
L= & -\frac{1}{n} \sum_{i=1}^{n}\left(y_{i} \log y_{i}^{\prime}+\left(1-y_{i}\right) \log \left(1-y_{i}^{\prime}\right)\right) \\
& +\lambda\|\Theta\|_{2}^{2}
\end{aligned}
$$

where $y_{i}^{\prime}$ is the predicted label for patient $i, \lambda$ is the weight parameter to balance two losses.

\section{Experiments and results}

\section{Dataset and experimental settings}

In this study, we evaluate our proposed model on a realworld clinical dataset consisting of 736 heart failure (HF) patients collected from the Cardiology Department at the Chinese PLA General Hospital. The objective of the experiments is to predict readmissions within 1 year based on the patient characteristics. Specifically, each patient contains 105 features, including demographics (e.g. age and gender), vital signs (e.g. blood pressure and heart rate), lab tests (e.g. NT-proBNP and CTnT), echocardiography (e.g. ejection fraction and QRS interval), comorbidities (e.g. diabetes and renal insufficiency), length of stay (LOS) and medications (e.g. ACEI/ARB, beta blocker and MRA). These patients were followed up for 1 year to check the readmissions within 1 year (461 readmitted, 275 not readmitted). Patient features with more than $30 \%$ missing values were not included in this work, while the features with less than $30 \%$ missing values were imputed by the median of the features.

Note that a prior ethics approval was obtained from the data protection committee and the institutional review board of the hospital, and the patient data was anonymized in our study.

We compared the performance of the proposed model with three baseline models: logistic regression (LR), MLP (without the attention mechanism) and stacked denoising auto-encoder (SDAE). For both the proposed and the baseline models, we employed the five-fold cross-validation strategy on $80 \%$ of the data for training and tuning the model, and evaluated the performance of the trained model on the rest $20 \%$ of the data (final test set) that was not used during the training process. The experiments were repeated ten times and the final performances were averaged on the ten repetitions. Accuracy, precision, recall, F1 score and AUC (Area under ROC curve) were employed as the evaluation metrics.

In terms of the hyper-parameter settings, the learning rate is 0.001 , the $\mathrm{L} 2$ coefficient $\lambda$ is 0.001 . The numbers of hidden layers of were tested from one to five.

\section{Prediction performance}

We firstly investigated the influence of the number of hidden layers of the proposed model on the performance of readmission prediction in terms of both accuracy and AUC, as illustrated in Fig. 2. The results show that the proposed model achieved the best performance in terms

Table 2 The $p$-value of paired t-test between the proposed model and baseline models

\begin{tabular}{lllll}
\hline Models & MLP_attention & MLP & LR & SDAE \\
\hline MLP_attention & - & 0.003 & 0.005 & 0.0008 \\
MLP & & - & 0.009 & 0.005 \\
LR & & - & 0.003 \\
SDAE & & & - \\
\hline
\end{tabular}


of both accuracy and AUC among the four models. With the number of hidden layers increasing, the performance of the proposed model decreased. For MLP, both accuracy and AUC reached the peak when there were four hidden layers, and began to drop when the number of hidden of layers increased to five probably because of over-fitting. SADE achieved the best performance with two encoding and decoding layers.

Table 1 records the performance of the proposed model (one hidden layer) and the three baseline models LR, MLP (four hidden layers) and SDAE (two encoding and decoding layers). It indicated that although MLP performed the best in terms of recall, the proposed model outperformed MLP, LR and SDAE in terms of accuracy, precision, F1 score and AUC. Specifically, with the attention mechanism, the proposed model had 2.5, 2.6, 1.1 and $1.2 \%$ performance gain compared to MLP in terms of accuracy, precision, F1 score and AUC respectively. This demonstrates that the attention mechanism can improve the performance of MLP.

To examine the statistical difference of the performances between the proposed model and the baseline models, we conducted the paired-samples t-test for each pair of models. The paired sample t-test is a statistical procedure used to determine whether the mean difference between two sets of observations is zero [29]. In our study, the proposed model and the baseline models predicted the labels (readmitted/non-readmitted) of all patient samples in the test sets and resulted in paired sets of observations. As can be seen in Table 2, the predicted labels of each pair of models are statistically different $(p<0.01)$.

\section{Attention analysis}

The interpretability of the generated prediction results is significantly important in healthcare. Since the proposed model is based on the attention mechanism, it is easy to obtain the contribution of each patient feature by the attention weights. Fig. 3 shows the heat map of the contribution (i.e., the attention weight) of each feature for readmission identified by the proposed model for 50 randomly selected patients. Each row is a patient sample and each column is a feature. Note that the 105 features are denoted as serial numbers in the heat map for clearness. The color in the heat map corresponds to the patient feature contribution (i.e. the log value of attention weight). From Fig. 3, we can observe that NT-proBNP (the 21st feature) got the most attention in almost all the patients. This observation is in accordance with the clinical practice, in which NT-proBNP is an essential risk factor for heart failure patients [30].

Additionally, we can observe the personalized risk factors for each patient besides NT-proBNP. For example, we present two heart failure patients with visualizing their relating predictors in Fig. 4. As we can see the first patient in Fig. 4a, Sodium (the 32nd feature) and CHD (Coronary Heart Disease) (the 12th feature) are the next two most important predictors, whilst for patient 2 in

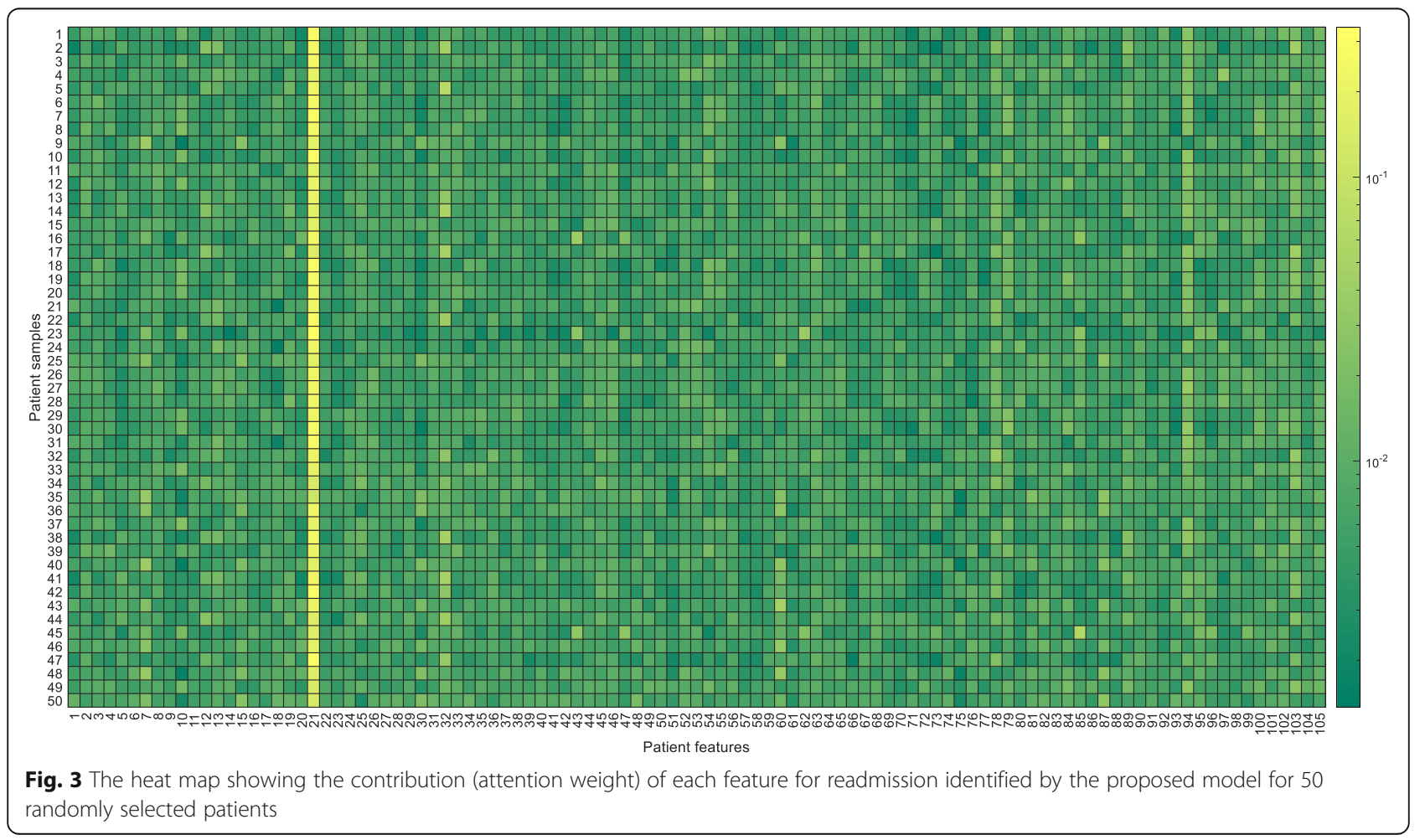




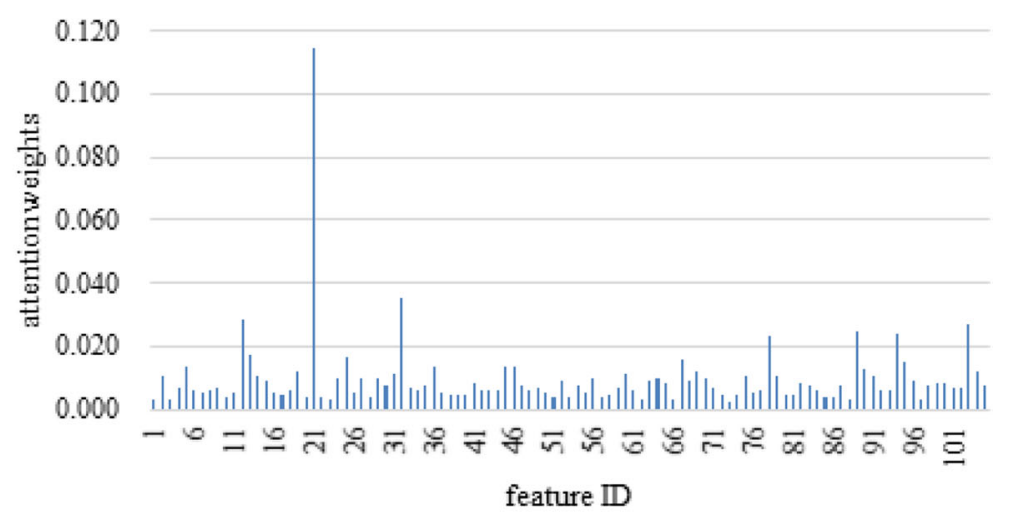

(a)

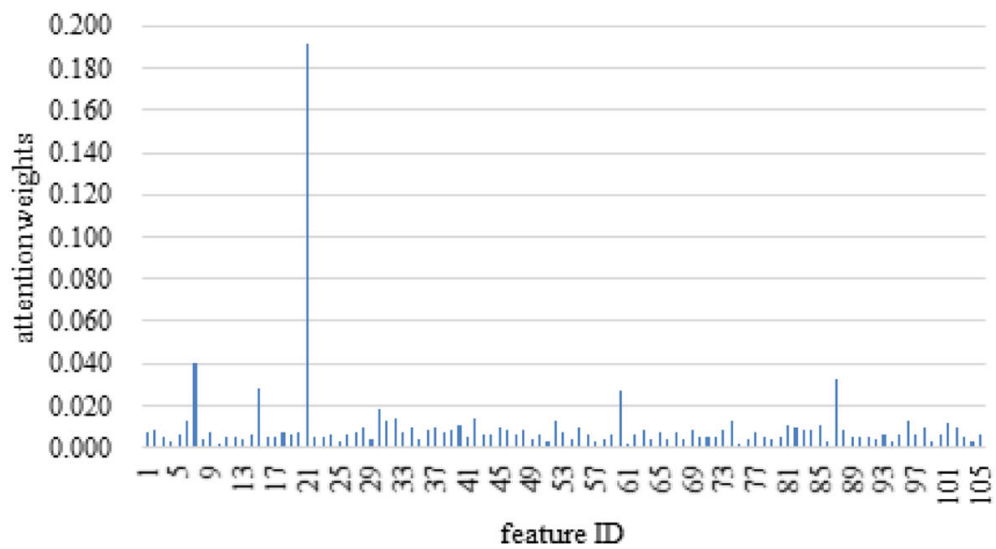

(b)

Fig. 4 The bar plots of the attention weights of each patient feature for two randomly selected patients

Fig. 4b, the next two most important predictors are $S B P$ (Systolic blood pressure) (the 7th feature) and Left ventricular end-systolic volume (the 87th feature). This shows that heart failure patient may have different subtypes, i.e. personalized profiles with individualized risk factors. In Table 3, we listed the top-ranked features (attention weights $>0.2$ ) of these two patients respectively. As the clinical guideline [30] denoted that many conditions or comorbidities are associated with the onset or development of HF and different patients may have different comorbidities, we can see that the important comorbidity for Patient 1 is CHD, while for Patient 2 diabetes is an important comorbidity. Besides, we can find that $C C B$ (calcium channel blocker) is identified as one of the top-ranked predictor of readmission for $\mathrm{Pa}$ tient 1 who were prescribed $C C B$, which is in line with the guidelines. According to the guideline [30], $C C B$ may be harmful and should be avoided to use in patients

Table 3 The top-ranked features of the two randomly selected patients in Fig. 4 (attention weights>0.2)

\begin{tabular}{|c|c|c|c|c|c|}
\hline \multicolumn{3}{|l|}{ Patient 1} & \multicolumn{3}{|l|}{ Patient 2} \\
\hline Feature ID & Name & Attention weights & Feature ID & Name & Attention weights \\
\hline 21 & NT-proBNP & 0.114 & 21 & NT-proBNP & 0.192 \\
\hline 32 & Sodium & 0.035 & 7 & SBP & 0.040 \\
\hline 12 & $\mathrm{CHD}$ & 0.028 & 87 & Left ventricular end-systolic volume & 0.033 \\
\hline 103 & Spironolactone & 0.027 & 15 & Diabetes & 0.028 \\
\hline 89 & Left ventricular end-diastolic volume index & 0.025 & 60 & Platelet count & 0.028 \\
\hline 94 & CCB & 0.024 & - & - & - \\
\hline 78 & Left atrial diameter & 0.023 & - & - & - \\
\hline
\end{tabular}


with low LVEF. The identified patient-specific risk factors could be further leveraged to assist the clinicians to customize the treatment strategies or intervention plans.

Moreover, the proposed model can not only provide individualized risk factors for each patient, but also provide the most frequent risk factors for all patient samples in a global sense. In Table 4, we listed the top-ten ranked features selected by both the proposed model and LR respectively. For the proposed model, the features are ranked by the frequency of the feature occurred in the top-ten features (ranking by attention weights) of each patient. For example, NT-proBNP is in the top-10 ranked features for all patients, while $C C B$ (calcium channel blocker) is in the top-10 ranked features for 374 patients. With respect to LR, the selected patient features are ranked by the absolute values of the coefficients of these features in the learning LR model. It can be seen that there are three features are the same for the two models, i.e. NT-proBNP, SBP (systolic blood pressure) and Platelet count, which have been reported to be predictive for the prognosis of HF in literature [30]. E.g., hypertension may be the single most important modifiable risk factor for HF, whilst elevated levels of diastolic and especially systolic blood pressure are major risk factors for the development of HF [30]. The findings in [31] demonstrated an association between thrombocytopenia (low platelet count) and increased mortality in patients with heart failure.

In addition to the three common risk factors, the proposed method identified three echocardiographic measurements in the top-ten ranked factors which were not identified by LR, i.e. Left atrial diameter, Interventricular septal thickness and Left ventricular end systolic volume. Echocardiography is used to evaluate the cardiac structure changes and left ventricular systolic function, and can help the clinicians make diagnosis and predict the risk of subsequent events (e.g. readmission) [30].

\section{Discussion}

From the experimental results, we have some interesting findings as follows:

- Combining Tables 1 and 2, our proposed model outperformed the baseline models MLP and LR statistically. It demonstrates that the attention mechanism can improve the performance of neural networks.

- From Fig. 2, we can see that our proposed model achieved better performance with less number of layers (one layer) than MLP (four layers). Note that less number of layers of a deep neural work may have lower computational cost during training. In addition, it is interesting that the performance of the proposed model drops with the increasing of the number of hidden layers. We plan to investigate this phenomenon in our future work.

- The attention mechanism can identify the patientspecific features related to the outcomes, which can not only help the domain experts understand the prediction outcomes, but also support the decision makers to make individualized decisions.

- We can also obtain the important feature in the population level by counting the frequency of the top-ranked features in all patients.

It should be mentioned that there exist some limitations in this study needed to be investigated in the future. In our proposed model, the attention weights for all the features are positive, which is not able to tell us whether the influence of the feature is positive or negative like LR. We plan to work on this issue in our future work. In addition, the dataset used in this study is small, while the deep learning models usually need large volume of data for training. We plan to validate the proposed model on larger datasets.

Table 4 The top-ten globally ranked features of the proposed model and LR

\begin{tabular}{|c|c|c|c|c|}
\hline \multicolumn{3}{|c|}{ MLP_attention } & \multicolumn{2}{|l|}{ LR } \\
\hline Feature ID & Name & Frequency & Feature ID & Name \\
\hline 21 & NT-proBNP & 736 & 21 & NT-proBNP \\
\hline 94 & $\mathrm{CCB}$ & 374 & 7 & SBP \\
\hline 78 & Left atrial diameter & 225 & 41 & Lactate dehydrogenase \\
\hline 103 & Spironolactone & 180 & 57 & Monocytes ratio \\
\hline 7 & SBP & 167 & 3 & Height \\
\hline 32 & Sodium & 146 & 8 & DBP (Diastolic blood pressure) \\
\hline 85 & Interventricular septal thickness & 146 & 5 & BMI \\
\hline 87 & Left ventricular end systolic volume & 140 & 34 & Phosphorus \\
\hline 10 & Anemia & 135 & 59 & Basophil ratio \\
\hline 60 & Platelet count & 126 & 60 & Platelet count \\
\hline
\end{tabular}




\section{Conclusions}

In this paper, we present an attention-based neural network model to improve the interpretability of the generated prediction results by the model. The patientspecific attention weights obtained from the model can not only help the clinicians understand the prediction outcomes, but also assist them to make further clinical decisions, such as customizing individualized treatment strategies or intervention plans for patients. We evaluated the proposed model on a real-world clinical dataset to address a specific clinical prediction problem, i.e., the readmission prediction for heart failure patients. The experimental results show that our proposed model outperforms the baseline models in terms of both accuracy and AUC.

Although our results have been encouraging in this study, the proposed approach could be further improved. At first, the dataset used in this study is relatively small, while the deep learning models usually need large volume of data for training. We plan to validate the proposed model on larger datasets in the future and compare the proposed model to more existing models. Besides, in this study, we simply exploited the numerical information for prediction. In our future work, we decided to utilize the abundant text data in the EHR (e.g. discharge notes, daily progress notes) to generate text interpretations.

\section{Abbreviations}

LR: Logistic regression; MLP: Multilayer perceptron; HF: Heart failure; NTproBNP: $\mathrm{N}$ terminal pro B type natriuretic peptide; $\mathrm{CTnT}$ : Cardiac troponin T; AUC: Area under ROC curve; CHD: Coronary heart disease; SBP: Systolic blood pressure; DBP: Diastolic blood pressure

\section{Acknowledgements}

Not applicable.

\section{About this supplement}

This article has been published as part of BMC Medical Informatics and Decision Making Volume 20 Supplement 3, 2020: Health Information Processing. The full contents of the supplement are available online at https:// bmcmedinformdecismak.biomedcentral.com/articles/supplements/volume-2 0-supplement-3.

\section{Authors' contributions}

All authors conceived of the proposed idea. PC planned the experiments, implemented the methods, carried out the experiments and evaluated the proposed models. PC and ZH wrote the manuscript with the comments from UK and XL. All authors have read and approved the final manuscript.

\section{Funding}

The publication cost is supported by the National Key Research and Development Program of China under Grant No. 2016YFC1300303, the National Natural Science Foundation of China under Grant No. 61672450, and Philips Research under the Brain Bridge Project. The publication costs for this manuscript were provided partly by the Grant No. 2016YFC1300303 and No. 61672450

\section{Availability of data and materials}

The datasets generated and/or analyzed during the current study are not publicly available due to the hospital's regulations, but are available from the corresponding author on reasonable request.
Ethics approval and consent to participate

Not applicable.

\section{Consent for publication}

Not applicable.

\section{Competing interests}

The authors declare that they have no competing interests.

\section{Author details}

${ }^{1}$ College of Biomedical Engineering and Instrumental Science, Zhejiang University, Hangzhou, China. ${ }^{2}$ School of Industrial Engineering, Eindhoven University of Technology, Eindhoven, The Netherlands. ${ }^{3}$ Department of Cardiology, Chinese PLA General Hospital, Beijing, China. ${ }^{4}$ Cardiocloud medical technology, Beijing, China.

Published: 9 July 2020

\section{References}

1. Shickel B, Tighe P, Bihorac A, Rashidi P. Deep EHR: A survey of recent advances in deep learning techniques for electronic health record (EHR) analysis. IEEE J Biomed Health. 2018;22(5):1589-604.

2. Xiao C, Choi E, Sun J. Opportunities and challenges in developing deep learning models using electronic health records data: a systematic review. J Am Med Inform Assoc. 2018:25(10):1419-28.

3. Esteva A, Robicquet A, Ramsundar B, et al. A guide to deep learning in healthcare. Nat Med. 2019;25(1):24

4. Huang $Z$, Dong $W$, Duan $H$, et al. A regularized deep learning approach for clinical risk prediction of acute coronary syndrome using electronic health records. IEEE Trans Biomed Eng. 2017:65(5):956-68.

5. Yang C, Delcher C, Shenkman E, et al. Predicting 30-day all-cause readmissions from hospital inpatient discharge data. In: 2016 IEEE 18th International conference on e-Health networking, applications and services (Healthcom); Munich: IEEE; 2016. p. 1-6.

6. Johansson F, Shalit U, Sontag D. Learning representations for counterfactual inference. In: International Conference on Machine Learning; 2016. p. 3020-9

7. Zhang Y, Chen R, Tang J, et al. LEAP: learning to prescribe effective and safe treatment combinations for multimorbidity. In: Proceedings of the 23rd ACM SIGKDD International Conference on Knowledge Discovery and Data Mining; New York: ACM; 2017. p. 1315-24.

8. Montavon G, Samek W, Müller KR. Methods for interpreting and understanding deep neural networks. Digit Signal Process. 2018;73:1-15.

9. Wang F, Kaushal R, Khullar D. Should health care demand interpretable artificial intelligence or accept "black box" medicine? Ann Intern Med. 2020; 172:59-60. [Epub ahead of print 17 December 2019].

10. Lipton ZC. The mythos of model interpretability. Commun ACM. 2018; 61(10):36-43.

11. Ribeiro MT, Singh S, Guestrin C. Why should I trust you? Explaining the predictions of any classifier. In: Proceedings of the 22nd ACM SIGKDD International Conference on Knowledge Discovery and Data Mining; New York: ACM; 2016. p. 1135-44.

12. Kim, Been. Interactive and interpretable machine learning models for human machine collaboration. PhD thesis, Massachusetts Institute of Technology, 2015

13. Huysmans J, Dejaeger K, Mues C, et al. An empirical evaluation of the comprehensibility of decision table, tree and rule based predictive models. Decis Support Syst. 2011:51(1):141-54.

14. Ahmad MA, Eckert C, Teredesai A. Interpretable machine learning in healthcare. In: Proceedings of the 2018 ACM International Conference on Bioinformatics, Computational Biology, and Health Informatics; New York: ACM; 2018. p. 559-60

15. Kaminski ME. The right to explanation, explained. Berkeley Tech $L J$. 2019;34:189.

16. Zhao J, Wang $T$, Yatskar $M$, et al. Men also like shopping: Reducing gender bias amplification using corpus-level constraints. In: Proceedings of the 2017 Conference on Empirical Methods in Natural Language Processing; 2017

17. Caruana R, Lou Y, Gehrke J, et al. Intelligible models for healthcare: Predicting pneumonia risk and hospital 30-day readmission. In: Proceedings 
of the 21th ACM SIGKDD International Conference on Knowledge Discovery and Data Mining; New York: ACM; 2015. p. 1721-30.

18. Kaymak U. On using fuzzy sets in healthcare process analysis. In: International Conference on Theory and Applications of Fuzzy Systems and Soft Computing. Cham: Springer; 2018. p. 24.

19. McAuley J, Leskovec J. Hidden factors and hidden topics: understanding rating dimensions with review text. In: Proceedings of the 7th ACM conference on Recommender systems; New York: ACM; 2013. p. 165-72.

20. Maaten L, Hinton G. Visualizing data using t-SNE. J Mach Learn Res. 2008; 9(Nov):2579-605.

21. Caruana R, Kangarloo H, Dionisio JD, et al. Case-based explanation of noncase-based learning methods. In: Proceedings of the AMIA Symposium; Washington, DC: American Medical Informatics Association; 1999. p. 212

22. Chen P, Dong W, Lu X, et al. Deep representation learning for individualized treatment effect estimation using electronic health records. J Biomed Inform. 2019;100:103303.

23. Bahdanau D, Cho K, Bengio Y. Neural machine translation by jointly learning to align and translate. arXiv preprint arXiv:1409.0473, 2014.

24. Vaswani A, Shazeer N, Parmar N, et al. Attention is all you need. In: Advances in Neural Information Processing Systems; 2017. p. 5998-6008.

25. Chorowski JK, Bahdanau D, Serdyuk D, et al. Attention-based models for speech recognition. In: Advances in Neural Information Processing Systems; 2015. p. $577-85$

26. Choi E, Bahadori MT, Song L, et al. GRAM: graph-based attention model for healthcare representation learning. In: Proceedings of the 23rd ACM SIGKDD International Conference on Knowledge Discovery and Data Mining; New York: ACM; 2017. p. 787-95.

27. Choi E, Bahadori MT, Sun J, et al. Retain: an interpretable predictive model for healthcare using reverse time attention mechanism. In: Advances in Neural Information Processing Systems; 2016. p. 3504-12.

28. Zhang X, Qian B, Li X, et al. An interpretable fast model for predicting the risk of heart failure. In: Proceedings of the 2019 SIAM International Conference on Data Mining; Calgary, Alberta, Canada: Society for Industrial and Applied Mathematics; 2019. p. 576-84.

29. Bland M. An introduction to medical statistics. UK: Oxford University Press; 2015.

30. Yancy CW, Jessup M, Bozkurt B, et al. 2013 ACCF/AHA guideline for the management of heart failure: a report of the American College of Cardiology Foundation/American Heart Association task force on practice guidelines. J Am Coll Cardiol. 2013;62(16):e147-239.

31. Mojadidi MK, Galeas JN, Goodman-Meza D, et al. Thrombocytopaenia as a prognostic indicator in heart failure with reduced ejection fraction. Heart Lung Circ. 2016;25(6):568-75.

\section{Publisher's Note}

Springer Nature remains neutral with regard to jurisdictional claims in published maps and institutional affiliations.

Ready to submit your research? Choose BMC and benefit from:

- fast, convenient online submission

- thorough peer review by experienced researchers in your field

- rapid publication on acceptance

- support for research data, including large and complex data types

- gold Open Access which fosters wider collaboration and increased citations

- maximum visibility for your research: over $100 \mathrm{M}$ website views per year

At $\mathrm{BMC}$, research is always in progress.

Learn more biomedcentral.com/submissions 